\title{
LA MODELIZACIÓN DEL HÁBITAT EN LA ESTIMACIÓN DE ABUNDANCIAA ESCALA LOCAL. LA PERDIZ ROJA EN UN AGROSISTEMA EN TOLEDO (ESPAÑA CENTRAL)
}

\author{
Javier García Garrido \\ Raúl Romero-Calcerrada \\ Carlos J. Novillo Camacho \\ Departamento de Tecnología Química y Ambiental. Universidad Rey Juan Carlos. Madrid \\ jgarcia@naturaliza.es, raul.romero.calcerrada@urjc.es
}

\section{RESUMEN}

El seguimiento de las poblaciones de fauna es un aspecto fundamental en los programas de gestión. En este estudio proponemos la utilización de un modelo de hábitat para la estimación de densidades de fauna (en nuestro caso de perdiz roja Alectoris rufa) a partir de los datos obtenidos en los muestreos de censo convencionales. Los resultados muestran que es posible mejorar la precisión de los resultados de censo, predecir los efectos del abandono de la actividad agrícola sobre la población de Alectoris rufa, y profundizar en los requerimientos ecológicos de la especie en el área de estudio.

Palabras clave: Modelos de hábitat, estimación de abundancia, estructura espacial del hábitat.

\section{ABSTRACT}

Tracking wildlife populations is an outstanding aspect on management programs. We propose in this study the use of habitat modeling methods to estimate fauna abundance (red partridge Alectoris rufa abundance in this case) from data obtained in conventional census

Fecha de recepción: septiembre 2012.

Fecha de aceptación: septiembre 2013. 
sampling. The results show that it is possible to improve Alectoris rufa census accuracy, to predict species population effects of drop farming activity, and to deepen the ecological requirements of Alectoris rufa in the study area.

Key words: Habitat modeling, abundance estimate, habitat spatial structure.

\section{INTRODUCCIÓN Y OBJETIVOS}

Las tareas de gestión a escala local, tales como la planificación cinegética, los planes de mejora agraria, y la ejecución de proyectos rurales, requieren un conocimiento exhaustivo del ecosistema involucrado de modo que se encuentren las alternativas más adecuadas para compatibilizar los aprovechamientos económicos con la conservación del medio. No obstante, en la mayor parte de las ocasiones no existen recursos suficientes para realizar estos estudios con el nivel de detalle necesario, lo que desemboca en una mala planificación y gestión. Concretamente, el seguimiento de las poblaciones de fauna es uno de los aspectos fundamentales en cualquier programa de gestión (Belda et al. 2011), de modo que se puedan establecer los efectos provocados por una determinada actividad o actuación a lo largo del tiempo. Generalmente, estos seguimientos son realizados a través de muestreos muy superficiales que en ocasiones no aportan la precisión requerida. En este sentido, los modelos de hábitat resultan una herramienta útil al ser capaces de predecir la distribución o la abundancia de una determinada especie (Guisan \& Zimmermann 2000 y Nielsen et al. 2005) cuando los datos de partida son escasos. Además, estos modelos de hábitat nos permiten realizar predicciones bajo unas condiciones diferentes a las actuales pudiendo predecir y valorar, por ejemplo, los efectos ocasionados por los cambios en el uso del suelo de una determinada actuación.

Durante los últimos años, el desarrollo de los modelos de hábitat potencial ha proporcionado una mejora en la capacidad de identificar zonas favorables para las especies (Pearce \& Ferrier 2000). Estos modelos interpolan o extrapolan las localizaciones donde se conoce la presencia de la especie mediante la relación de estos lugares con variables ambientales (Pearce \& Boyce 2006), dando como resultado un mapa de probabilidad que refleja el grado de adecuación del hábitat para todo el territorio analizado.

Normalmente, estos modelos de hábitat son utilizados para predecir tanto la distribución de especies raras o amenazadas como la dispersión potencial de una especie invasora (Engler et al. 2004), tratándose siempre de especies con poblaciones reducidas o hábitats restringidos. Sin embargo existen pocos estudios sobre especies ampliamente extendidas y en los estudios realizados se han obtenido resultados contradictorios en cuanto a la relación existente entre ocurrencia y abundancia (Nielsen et al. 2005). Del mismo modo, este tipo de modelos de hábitat son generalmente utilizados para predecir la distribución de una determinada especie a escalas regionales o continentales que abarcan amplias extensiones geográficas, para lo cual se emplean principalmente variables topográficas y/o climáticas no teniéndose en cuenta ningún tipo de variable con aspectos biológicos o ecológicos (Araújo \& Louto 2007). No obstante, para estudios a escala local lo más adecuado es utilizar variables relacionadas con las características del hábitat que se relacionen con los requerimientos de la especies a estudiar (Romero-Calcerrada \& Luque 2006). Además, teniendo en cuenta que hoy en día se producen cambios muy rápidos en los usos del suelo que ocasionan la frag- 
mentación del hábitat (Belda et al. 2011), se hace necesario la utilización de índices sobre la estructura del hábitat que representen e indiquen la fragmentación del mismo, de modo que sea posible predecir los efectos producidos por determinadas actuaciones.

La planificación y gestión cinegética representa un caso concreto de gestión local en el cual resulta necesario la realización de un seguimiento de las poblaciones de fauna de forma exhaustiva. En la comarca estudiada del norte de la provincia de Toledo, la especie cinegética que tradicionalmente ha poseído una mayor importancia económica debido a su abundancia es la Alectoris rufa (perdiz roja). Además, y más importante si cabe, esta especie supone un elemento vital en la cadena trófica de la fauna mediterránea (Blanco Aguiar et al. 2004), siendo importante mantener sus poblaciones para la conservación de otras especies depredadoras (reptiles, mamíferos y rapaces como p.e. elAzor común, el Águila calzada, el Águila perdicera y el Águila real). No obstante, la especie Alectoris rufa se caracteriza por estar muy ligada al medio agrario (Fortuna 2002), lo que supone que sus poblaciones se vean rápidamente afectadas por los cambios de ocupación del suelo, especialmente en la agricultura. En este sentido, sus principales amenazas han sido históricamente la sobreexplotación cinegética y el abandono o intensificación agraria, lo que ha provocado la transformación del hábitat (pérdida de linderos y homogeneización del paisaje agrícola) (Blanco Aguiar et al. 2004).

Los objetivos de este estudio son los siguientes: (1) determinar si la utilización de un modelo de hábitat a escala local supone una mejora en la estimación de abundancias de Alectoris rufa; (2) definir la contribución que han podido tener los cambios de ocupación del suelo sobre la población de Alectoris rufa; (3) contribuir al conocimiento de los requerimientos de hábitat de Alectoris rufa en un agrosistema de la zona centro de la Península Ibérica.

\section{II. ÁREA Y ESPECIES OBJETO DE ESTUDIO}

\section{1. Área de estudio}

El estudio se ha realizado sobre el territorio delimitado por el coto privado de caza de la asociación de cazadores del municipio de Valmojado (Toledo). Este coto de caza se ubica al norte de la provincia de Toledo, englobando el término municipal de Valmojado, y parcialmente el de Casarrubios del Monte y Méntrida (Figura 1). La superficie total ocupada es de 6.758 ha. Según los datos del Padrón de 2010, la población supera los 14.000 habitantes en el conjunto de los tres municipios, y la densidad de población es de 0,70 habitantes por hectárea.

Se trata de una zona con una geomorfología ondulada, con altitudes comprendidas entre los 550 y 680 m.s.n.m. Dentro de esta tipología geomorfológica se distinguen claramente dos zonas; la zona sur es más llana, mientras que la zona norte queda formada por pequeños valles y lomas. El clima queda definido por el piso bioclimático supramesomediterráneo (Rivas Martínez 1987), presentando periodos largos de sequía y heladas. La pluviometría anual es de unos $500 \mathrm{~mm}$, y la evapotranspiración de $750 \mathrm{~mm}$, por lo que por lo general se produce un déficit hídrico durante los meses de estío. Las diferentes zonas existentes en el área de estudio presentan un clima muy homogéneo a pesar de sus diferencias geomorfológicas, por lo que no se distinguen variaciones climáticas significativas. 


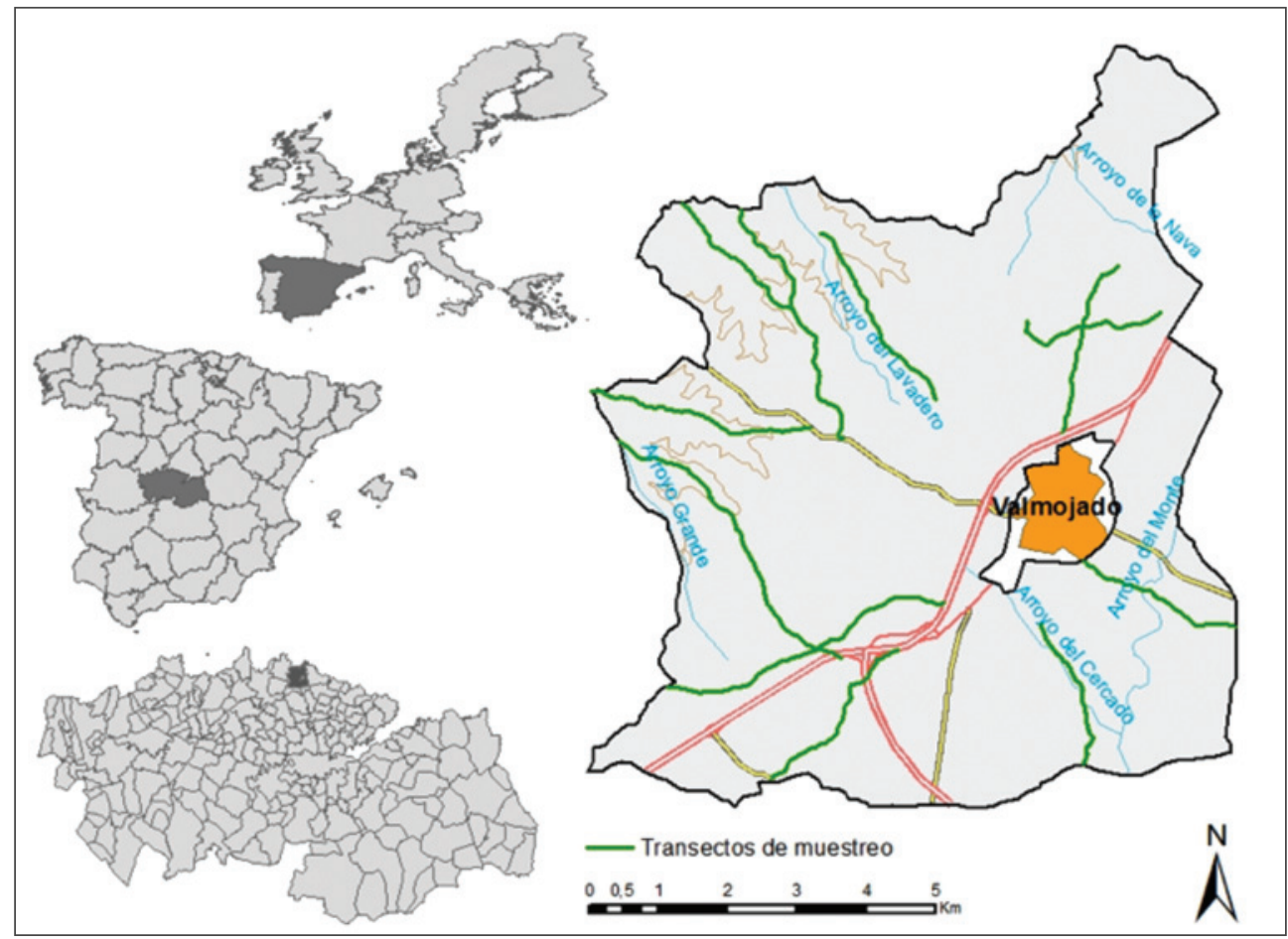

Este territorio constituye una buena representación del paisaje agrícola tradicional. Su característica principal es la estructura en mosaico de cultivos de viñedo y cereal de secano, con la inclusión de olivares de reducido tamaño, y algunas manchas muy aisladas de monte mediterráneo en aquellas áreas menos accesibles o menos fértiles. La zona norte presenta mayores inclusiones de vegetación natural, mientras que la sur ostenta una faceta más agrícola. No obstante la fragmentación del territorio es elevada en ambas zonas como consecuencia del reducido tamaño de las parcelas agrícolas, cuyo tamaño medio en el área de estudio es de tan sólo 0,74 hectáreas por parcela (Fuente: Catastro de Rústica, 2012). Por otro lado, una característica común en el área de estudio es el abandono de tierras agrícolas que se viene produciendo durante los últimos años, provocando una transformación de este mosaico de cultivos con un aumento de la superficie de pastos y formaciones arbustivas. El censo agrario realizado en los años 1999 y 2009 denota este abandono de la actividad agrícola (Figura 2), apreciándose una disminución en la superficie dedicada a los principales cultivos agrícolas (herbáceos, olivar y viñedo) y un aumento de la superficie destinada a pastos.

Este tipo de agro-sistemas es capaz de albergar altas densidades de especies cinegéticas de caza menor (Belda et al. 2011), tratándose de hecho de una de las áreas cinegéticas de caza menor más importantes de la zona centro peninsular. Además, la alta densidad de especies cinegéticas de caza menor favorece la presencia de otras especies de fauna, princi- 


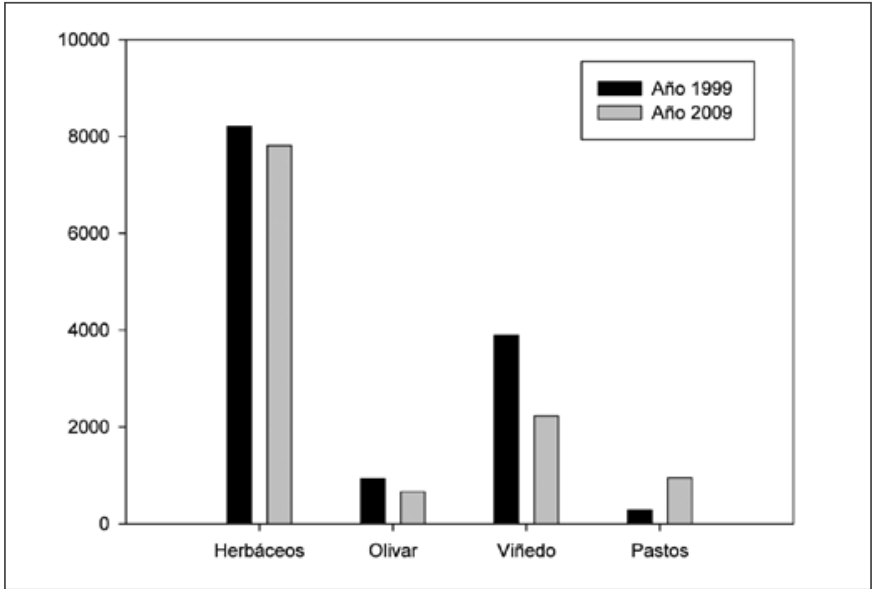

palmente predadoras. Toda la superficie de estudio presenta una gestión cinegética homogénea, la cual ha sido llevada a cabo sin ningún tipo de variación durante los últimos 10 años manteniéndose las modalidades de caza y el número de capturas autorizadas durante todo el periodo. En esta planificación cinegética las principales especies objeto de aprovechamiento son Oryctolagus cuniculus (conejo), Lepus granatensis (liebre ibérica), Alectoris rufa (perdiz roja), zorzales y palomas. De todas ellas Alectoris rufa es la especie de la que se obtiene un mayor aprovechamiento mediante su caza en la modalidad de «ojeo». A pesar de la utilización de esta modalidad de caza, el coto se ha caracterizado durante los últimos 10 años por sostener una regeneración natural de sus poblaciones de Alectoris rufa de forma anual, no siendo necesario el refuerzo demográfico mediante suelta de individuos procedentes de granjas. Este hecho hace que podamos considerar como estable y equilibrada la población de Alectoris rufa, a pesar de la presión cinegética que se ejerce sobre la especie, lo que nos permite tratar de modelizar con fiabilidad su hábitat idóneo.

\subsection{La especie cinegética objeto de estudio: Alectoris rufa}

Como especie objeto de estudio se ha seleccionado Alectoris rufa por ser la especie cinegética más abundante y más representativa en este territorio. Además se trata de una especie muy ligada a la estructura del paisaje y la ocupación del suelo (Buenestado et al. 2008), pudiendo presentar dentro un mismo territorio de reducido tamaño una importante variabilidad en su abundancia en función de dichos factores. Por otro lado, esta especie tiene una gran repercusión económica sobre la zona como consecuencia de su aprovechamiento cinegético, motivo por el cual es necesario llevar a cabo una adecuada gestión del recurso, tanto para garantizar la conservación del ecosistema como para mantener el aprovechamiento cinegético.

Alectoris rufa es una especie endémica del suroeste de Europa, estando presente en toda la Península Ibérica. Su distribución está asociada a áreas agrícolas, con espacios abiertos 
y sistemas de explotación en extensivo (Lucio \& Purroy 1992). En los agrosistemas de la zona centro de España la especie Alectoris rufa selecciona hábitats con abundantes linderos provistos de vegetación arvense y con manchas de matorral mediterráneo dispersas entre los cultivos (Fortuna 2002 y Tapia \& Domínguez 2007), proporcionándoles dicha composición refugio y alimento. En este sentido, la diversidad y estructura paisajística es un factor relevante en la selección del hábitat (Blanco Aguiar et al. 2004). Su área de campeo varía en función de las características del hábitat y de la actividad humana, situándose en mosaicos de cereal, viñedo y olivar del centro peninsular entre 20 y 30 ha (Buenestado et al. 2008). En relación a su abundancia, las densidades reproductoras varían de 5 a 20 parejas por cada 100 ha, y las densidades en la época previa a la caza entre 11 y 100 perdices por cada 100 ha. Estas poblaciones más densas se sitúan en el centro y en el sur de la Península, y especialmente son muy abundantes en el norte de Toledo, sureste de Ciudad Real y suroeste de Albacete. No obstante, las poblaciones de Alectoris rufa han sufrido un declive generalizado desde la década de los sesenta (Blanco Aguiar et al. 2004).

\section{MÉTODOS}

\subsection{Muestreo de presencia de Alectoris rufa}

El muestreo ha sido diseñado para obtener los datos necesarios para la estimación de la densidad de Alectoris rufa, y para poder llevar a cabo la modelización de su hábitat. Para ello se han realizado transectos de longitud variable (entre 2 y $5 \mathrm{~km}$ ) a través de los caminos existentes en el área de estudio. El esfuerzo de muestreo se estableció en $1 \mathrm{~km}$ de muestreo por cada 200 ha de superficie de estudio, por lo que de entre todos los caminos existentes en el coto se seleccionaron aleatoriamente 11 itinerarios alcanzando una longitud total de 37,57 $\mathrm{km}$ (Figura 1). Estos itinerarios se llevaron a cabo en coche a una velocidad de entre 10-20 $\mathrm{km} / \mathrm{h}$ durante las tres primeras horas tras el amanecer, con buenas condiciones climáticas, y siguiendo un procedimiento similar al llevado a cabo por Borralho et al. (1996). En cada transecto se registraron los ejemplares de Alectoris rufa detectados a ambos lados de la línea de progresión, anotando la distancia en perpendicular al ejemplar en base a cuatro bandas preestablecidas paralelas al transecto hasta 100 m de distancia máxima (Emlen 1977, Tellería 1986). Además, también se anotaron las coordenadas UTM desde donde se produce el contacto.

El muestreo se llevo a cabo durante la primera semana de noviembre de 2011. Se eligió esta fecha por dos factores: en primer lugar porque se trata de un periodo inicial de la temporada de caza, cuando la densidad de Alectoris rufa adulta presenta sus valores más altos; y en segundo lugar porque durante esta época ya han nacido y rebrotado tanto la hierba como las siembras de cereal, por lo que no existe escasez de alimento que pudiera sesgar la distribución hacia alguna fuente de alimento concreta.

\subsection{Descripción de las variables explicativas}

Las variables ambientales que van a ser utilizadas en el modelo de hábitat han de representar la variabilidad ecológica que posee el área de estudio en relación a los parámetros que determinan la presencia de la especie. Por lo general se asume que los procesos ecológicos 
que ocurren a escala local son los que definen las diferentes poblaciones y comunidades (Belda et al. 2011), mientras que las propias características ecológicas de la especie Alectoris rufa también indican directamente su relación con la estructura y composición del ecosistema a escala local. En consecuencia, para representar este tipo de parámetros a escala local del área de estudio se ha considerado utilizar tres tipos de variables: variables topográficas, usos del suelo, e índices sobre la estructura del paisaje. Todo el procedimiento llevado a cabo para elaboración de cada una de las variables queda representado en el diagrama de la Figura 3.

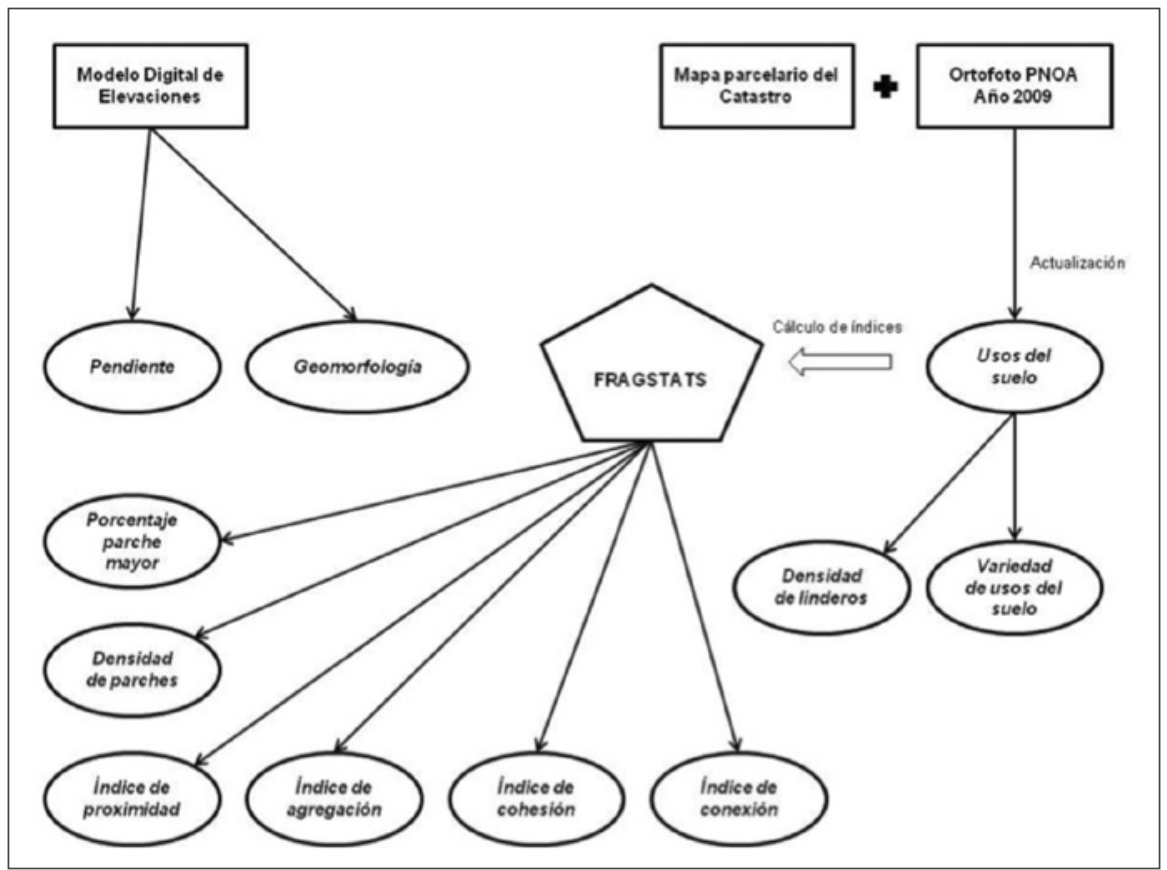

Las variables topográficas han sido obtenidas a partir del modelo digital de elevaciones (MDE) generado por el proyecto PNOA (Plan Nacional de Ortofotografía Aérea), el cual es llevado a cabo por el Ministerio de Fomento. Este MDE ha sido procesado mediante el software Esri® ${ }^{\circledR}$ Arcmap $^{\mathrm{TM}} 10.0$ y la herramienta «Topographic Position Index» creada por Jenness (2006) para la obtención de las variables pendiente y geomorfología.

La cartografía vectorial del catastro de rústica de la Dirección General del Catastro se ha utilizado para la obtención de los usos del suelo. Se optó por utilizar esta cartografía debido a que se trata de una información de libre acceso que aporta detalles muy precisos sobre la parcelación y consecuente fragmentación del territorio, los cuales son difíciles de conseguir a través de otras bases de datos. El inconveniente que presenta esta cartografía reside en que 
los usos del suelo inventariados en la base de datos no se encuentran muy actualizados. Por tanto, puesto que se trata de una zona donde durante los últimos años se está produciendo un abandono continuo de tierras agrícolas, fue necesario realizar una actualización de estos usos. Para realizar dicha actualización se utilizó una ortofoto aérea del proyecto PNOA correspondiente al año 2009. Sobre esta ortofoto se realizó una clasificación no supervisada tratando de distinguir diferentes grados de cobertura vegetal, los cuales sirvieron posteriormente para identificar aquellas parcelas agrícolas que habían sido abandonadas y colonizadas por vegetación natural. Posteriormente, mediante la aplicación de una fórmula de condicionalidad aquellas parcelas agrícolas donde el grado de cobertura vegetal era alto fueron transformadas a los usos «pastizal» o «pasto arbustivo». Una vez realizado el análisis, se consideró que los usos del suelo sin actualizar definen el estado del territorio en el año 2000, puesto que este es el año de revisión del parcelario del catastro; mientras que los usos del suelo actualizados definen el territorio en el año 2009, si bien para la modelización se han utilizado datos de muestreo obtenidos en el 2011 ya que se consideró que los cambios producidos durante estos dos años no eran significativos.

Los índices sobre la estructura del paisaje fueron obtenidos a partir de la variable usos del suelo, tanto para el año 2009 como para el año 2000, obteniendo así dos grupos de variables que definen las características del territorio en cada uno de esos años. A partir de estas dos capas de usos del suelo se realizaron análisis con los programas Fragstats® (McGarigal et al. 2002) y Esri ${ }^{\circledR}$ Arcmap $^{\mathrm{TM}}$ 10.0, obteniendo una serie de variables que explican parámetros de fragmentación y estructura del paisaje. Para el cálculo de estos índices se estableció un área de análisis circular de $250 \mathrm{~m}$ de radio en todos los casos, considerando dicho área como el área media de campeo de las perdices en esta zona (Buenestado et al. 2008). Dentro de todos los índices incluidos en el programa Fragstats ${ }^{\circledR}$, se seleccionaron únicamente aquellos índices que analizan la estructura del paisaje en su conjunto, y de éstos, se trató de elegir los índices más adecuados sobre la densidad y tamaño de los parches, su nivel de agregación, y la conectividad y proximidad existente entre ellos. Como resultado se seleccionaron 6 índices para nuestro estudio, los cuales ya han sido utilizados en otros estudios similares sobre paisajes agrarios (Billeter 2008, Pôças 2011).

Complementariamente, con el programa Esri ${ }^{\circledR}$ Arcmap $^{\mathrm{TM}} 10.0$ se crearon las variables densidad de linderos y la variedad de clases. Estas dos variables no fueron calculadas con Fragstats ${ }^{\circledR}$ debido a que dicho programa no calcula estos índices como tal, considerando sólo linderos aquellos límites existentes entre clases diferentes, mientras que nuestra zona de estudio presenta linderos con vegetación natural entre la mayor parte de las parcelas agrícolas, independientemente de que se trate de clases iguales o diferentes. Por tanto, al tratarse de un factor relevante para la ecología de la especie Alectoris rufa, estas variables fueron calculadas directamente a partir de la capa vectorial del parcelario del catastro, teniendo en cuenta así todos los linderos realmente existentes.

En su conjunto se obtuvieron un total de 8 índices de estructura del paisaje, dos variables topográficas, y una que representa los usos del suelo (Tabla 1). Especialmente los 8 índices que representan la estructura del paisaje han sido creados para tratar de representar la máxima variabilidad del ecosistema y permitir un mejor ajuste del modelo de hábitat, pero al tratarse de índices muy similares en algunos casos, puede que exista una correlación relevante entre algunos de estos índices. No obstante existen técnicas de modelización estables 
a la multicolinealidad (Elith et al, 2011), por lo que es posible su utilización, pero es un aspecto que puede influir significativamente en la interpretación biológica de los resultados.

Tabla 1

VARIABLES UTILIZADAS PARA LA MODELIZACIÓN DEL HÁBITAT DE LAALECTORIS RUFA

\begin{tabular}{|c|c|c|}
\hline Tipo de variable & Variable & Definición \\
\hline \multirow{2}{*}{ Topográficas } & Pendiente & $\begin{array}{l}\text { Gradiente de la diferencia de cota en grados. } \\
\left.\text { (Esri }{ }^{\circledR} \text { Arcmap }{ }^{\mathrm{TM}} 10.0\right) \text {. }\end{array}$ \\
\hline & Geomorfología & $\begin{array}{l}\text { Clasificación de las forma del terreno en tres } \\
\text { categorías: valles, cerros, y zonas intermedias. } \\
\text { (Jenness, 2006). }\end{array}$ \\
\hline $\begin{array}{l}\text { Usos y ocupación } \\
\text { del suelo }\end{array}$ & $\begin{array}{l}\text { Usos y ocupación } \\
\text { del suelo }\end{array}$ & $\begin{array}{l}\text { Clasificación en } 6 \text { categorías: } 1 \text { (cereal secano), } \\
2 \text { (viñedo), } 3 \text { (olivar), } 4 \text { (pastizal), } 5 \text { (pasto } \\
\text { arbustivo) y } 6 \text { (improductivo). }\end{array}$ \\
\hline \multirow{8}{*}{$\begin{array}{l}\text { Índices sobre la } \\
\text { estructura del } \\
\text { paisaje }\end{array}$} & $\begin{array}{l}\text { Variedad de usos } \\
\text { y ocupación }\end{array}$ & $\begin{array}{l}\text { Número total de ocupaciones diferentes en el } \\
\left.\text { radio de análisis. (Esri }{ }^{\circledR} \text { Arcmap }{ }^{\mathrm{TM}} 10.0\right) \text {. }\end{array}$ \\
\hline & $\begin{array}{l}\text { Densidad de } \\
\text { parches }\end{array}$ & $\begin{array}{l}\text { Número de parches por unidad de área (ha) en el } \\
\text { radio de análisis (Fragstats@ } 3.3 \text { ) }\end{array}$ \\
\hline & $\begin{array}{l}\text { Densidad de } \\
\text { linderos }\end{array}$ & $\begin{array}{l}\text { Número de linderos por unidad de área (ha), } \\
\text { calculado mediante la función kernel. (Esri® } \\
\text { Arcmap }^{\mathrm{TM}} \text { 10.0). }\end{array}$ \\
\hline & $\begin{array}{l}\text { Porcentaje parche } \\
\text { mayor }\end{array}$ & $\begin{array}{l}\text { Porcentaje de superficie ocupada por el parche } \\
\text { más grande dentro del radio de análisis. } \\
\text { (Fragstats@ } 3.3 \text { ) }\end{array}$ \\
\hline & $\begin{array}{l}\text { Índice de } \\
\text { proximidad }\end{array}$ & $\begin{array}{l}\text { Relación entre el tamaño de los parches y la } \\
\text { proximidad entre parches en el radio de análisis } \\
\text { (Fragstats® 3.3) }\end{array}$ \\
\hline & $\begin{array}{l}\text { Índice de } \\
\text { agregación }\end{array}$ & $\begin{array}{l}\text { Relación entre la homogeneidad existente y la } \\
\text { homogeneidad máxima posible en el radio de } \\
\text { análisis (Fragstats } ® 3.3 \text { ) }\end{array}$ \\
\hline & $\begin{array}{l}\text { Índice de } \\
\text { cohesión }\end{array}$ & $\begin{array}{l}\text { Medida de la conexión física existente para cada } \\
\text { tipo de uso en el radio de análisis (Fragstats }{ }^{\circledR} \\
3.3 \text { ) }\end{array}$ \\
\hline & $\begin{array}{l}\text { Índice de } \\
\text { conexión }\end{array}$ & $\begin{array}{l}\text { Número de conexiones existentes entre un tipo } \\
\text { de usos en función de una distancia especificada, } \\
\text { que en este caso es } 250 \mathrm{~m} \text {. (Fragstats }{ }^{\circledR} \text { 3.3) }\end{array}$ \\
\hline
\end{tabular}




\subsection{Modelización de hábitat}

Existen diversas técnicas de modelización del hábitat, aunque todas presentan diferentes limitaciones que condicionan su aplicabilidad (Elith et al, 2006; Mateo et al, 2011). Por ello, en la actualidad la tendencia es la utilización de modelos de consenso (Araújo \& New, 2007), los cuales integran los resultados obtenidos mediante diferentes técnicas de modelización con el fin de minimizar dichas limitaciones. Sin embargo, en este caso se ha optado por la utilización de un único modelo de sólo presencias, considerando que esta metodología es mucho más simple en su utilización e interpretación de los resultados, se adaptaba bien a los datos recogidos en el muestreo realizado, y se obtiene una precisión y fiabilidad suficientes para la gestión cinegética y del hábitat a la que va dirigida el análisis. Entre los diversos modelos existentes que únicamente utilizan datos de presencia, como ENFA (Análisis de Nicho Ecológico) (Hirzel et al. 2002), GARP (Genetic Algorithm for Rule-set Production) (Stockwell \& Peters 1999) o MaxEnt (Phillips et al. 2006), se ha seleccionado este último. El motivo ha sido que algunos de los trabajos comparativos realizados coinciden en que la interpretación de las representaciones de MaxEnt es mejor y más sencilla (Sérgio et al. 2007, Benito de Pando \& Peñas de Giles 2007) a la de los métodos ENFA y GARP, y que se trata de un método bastante estable en la utilización de variables correlacionadas (Elith et al, 2011).

La idea básica de MaxEnt es estimar la probabilidad de distribución de una especie, y la mejor estimación es asegurar que la aproximación tiene entropía máxima (Phillips et al. 2006). La entropía es definida por Shannon (1948) como cuánto la «elección» está implicada en la selección de un acontecimiento, por lo que la máxima entropía se refiere a la máxima elección. La técnica obliga a la modelización a emparejar ciertas características (variables ambientales) con los datos empíricos (datos de presencia de la especie a estudiar) y a elegir la condición de probabilidad que satisface que estas restricciones sean tan uniformes como sea posible (Buehler \& Ungar, 2001). Las principales limitaciones de este tipo de modelos son (1) que se asume que la población está en equilibrio con las condiciones ambientales, (2) que no se tienen en cuenta las relaciones bióticas, (3) que no se tienen en cuenta las relaciones espaciales del territorio, y (4) que en el caso de especies generalistas los resultados son poco fiables si no se utiliza un numero abundante de presencias (Mateo et al, 2011). La primera de las limitaciones se ha tratado de solventar realizando el muestreo al inicio de la temporada cinegética, cuando tras ocho meses de inactividad cinegética las poblaciones de Alectoris rufa se han restablecido y presentan un mayor equilibrio. Las relaciones bióticas es un parámetro difícil de controlar, aunque en este caso se ha tenido en cuenta la disponibilidad de alimento y agua realizando el muestreo en una época en la que dichos recursos no son limitantes. Las relaciones espaciales se han tenido en cuenta a través de los índices de estructura del hábitat utilizados como variables dependientes. Y por último, para tratar de obtener un modelo lo más fiable posible se ha utilizado un número relativamente elevado de presencias (73) en relación con el reducido área de estudio (6.758 has), con el objeto de que estas presencias utilizadas representen toda la variabilidad ecológica de la especie y permitan modelizar el hábitat más idóneo.

Utilizando como variable independiente cada una de las observaciones obtenidas en el muestreo y como variables dependientes todas las variables ambientales generadas, se obtuvo un modelo de hábitat para la especie Alectoris rufa. Las localizaciones exactas de 
cada una de las observaciones se obtuvieron a partir de las coordenadas UTM del punto de observación y de la distancia en perpendicular al ejemplar avistado. Por otro lado, los parámetros de configuración del programa se dejaron por defecto, a excepción del número de muestras a utilizar en el test que se estableció en una selección aleatoria del 25\% del total de las localizaciones. Como formato de salida del resultado se eligió formato logístico, lo que genera un mapa de probabilidad con valores comprendidos entre 0 y 1 fácilmente interpretables. Obtenido el resultado inicial del modelo se procedió a su ajuste mediante el análisis de la curva ROC, eliminando aquellas variables que tenían un efecto negativo sobre dicha curva para las localizaciones utilizadas en el test.

Una vez realizado este modelo inicial que refleja la situación actual, se procedió a realizar una proyección del modelo al año 2000 mediante la utilización de las variables ambientales correspondientes a dicho año.

\subsection{Estimación de la abundancia}

Se realizaron cálculos de densidad de Alectoris rufa mediante dos metodologías: en primer lugar considerando un único estrato; y en segundo lugar dividiendo el territorio en estratos tomando como diferentes estratos la clasificación de adecuación del hábitat obtenida a partir del modelo generado. No obstante en ambos casos se utilizó para el cálculo el programa Distance 6.0 Release 2 (Thomas et al. 2010). En consecuencia, la densidad ha sido estimada a partir de la distancia perpendicular a los ejemplares detectados, teniendo en cuenta los siguientes supuestos (Buckland et al. 2001): 1) Los objetos son detectados con certeza; 2) Los objetos no se mueven antes de que sea anotada la distancia perpendicular; 3) Las distancias entre el punto de observación y el objeto son precisas. Para la ejecución de los cálculos se utilizó el tipo de análisis denominado «CDS» (Conventional distance sampling).

Para poder realizar el cálculo de densidad por estratos, en primer lugar fue necesario reclasificar el modelo de hábitat obtenido para poder establecer las superficies correspondientes a cada uno de los estratos, definir los muestreos realizados en cada uno de los estrados, y contabilizar el número de observaciones efectuadas en cada uno de estos muestreos. El modelo fue reclasificado en dos clases o estratos utilizando el umbral que iguala la sensibilidad con la especificidad, punto a partir del cual es necesario aumentar mucho el área predicho para obtener una pequeña reducción del ratio de omisión. Una vez reclasificado el modelo se realizó un filtro para eliminar porciones de territorio con un tamaño demasiado pequeño como para considerarse un estrato diferenciado. Para ello se utilizó como área de análisis una superficie circular de $250 \mathrm{~m}$ de radio, considerando esta superficie como la superficie necesaria mínima con unas mismas condiciones ambientales para la supervivencia de Alectoris rufa en esta zona (en base al área de campeo estimado por Buenestado et al. 2008).

\section{RESULTADOS}

\subsection{Muestreo de Alectoris rufa}

La realización del recorrido de $37,57 \mathrm{~km}$ correspondiente a los transectos del muestreo dio como resultado un total de 73 observaciones directas de Alectoris rufa. Todas estas obser- 
vaciones se realizaron en 9 de los 11 transecos establecidos, por lo que en dos de ellos no se efectuó ninguna observación. En el resto de transectos el número de observaciones varió entre 1 y 18 . Como media se obtuvo un ratio de observación en el muestreo de 1,92 ejemplares por cada $\mathrm{km}$ de transecto recorrido.

\subsection{Características del hábitat}

Con el modelo de hábitat realizado se obtuvo la probabilidad de presencia de Alectoris rufa en función de las variables reflejadas en la Tabla 2 las cuales contribuyeron al modelo según el porcentaje indicado. Seleccionando estas 9 variables de las 11 disponibles, se obtuvieron unos valores de AUC de 0,827 para los datos de entrenamiento y 0,784 para los datos de evaluación (Figura 4). Estos valores obtenidos no son muy altos debido a que se trata de una especie muy extendida por toda el área del estudio, pero la igualdad entre el valor correspondiente a los datos de entrenamiento y el valor correspondiente a los datos de evaluación indica un alto poder de predicción. Las cuatro variables que más contribuyeron al modelo (sumando entre ellas un $65,5 \%$ de contribución), indicaron que las perdices prefieren fondos de valle frente a cerros o zonas de ladera; que evitan la presencia de parches muy grandes en su área de campeo (especialmente a partir de las 70 ha) y seleccionan aquellas áreas con alta densidad de parches; y en cuanto a la ocupación del suelo, a pesar de no tratarse de un factor limitante por su nivel de contribución al modelo se observó una preferencia por los viñedos y un ligero rechazo a los pastizales (los cuales se corresponden en su gran mayoría con tierras agrícolas actualmente abandonadas). No obstante al existir una alta correlación entre los diferentes índices de estructura del paisaje, no es posible precisar con exactitud cuál de los parámetros descritos es el que en mayor grado determina el hábitat potencial de la especie.

Tabla 2

PORCENTAJE DE CONTRIBUCIÓN DE CADA UNA DE LAS VARIABLES AMBIENTALES AL MODELO

Variable

Porcentaje de contribución al modelo

\begin{tabular}{lc}
\hline Geomorfología & 19,9 \\
Porcentaje de parche mayor & 19,5 \\
Densidad de parches & 14,5 \\
Usos del suelo & 11,5 \\
Índice de conexión & 8,2 \\
Variedad de usos del suelo & 7,7 \\
Pendiente & 7,6 \\
Densidad de linderos & 6,4 \\
Índice de cohesión & 4,4
\end{tabular}


Figura 4

CURVA ROC CORRRESPONDIENTE AL MODELO DE HÁBITAT DE ALECTORIS RUFA

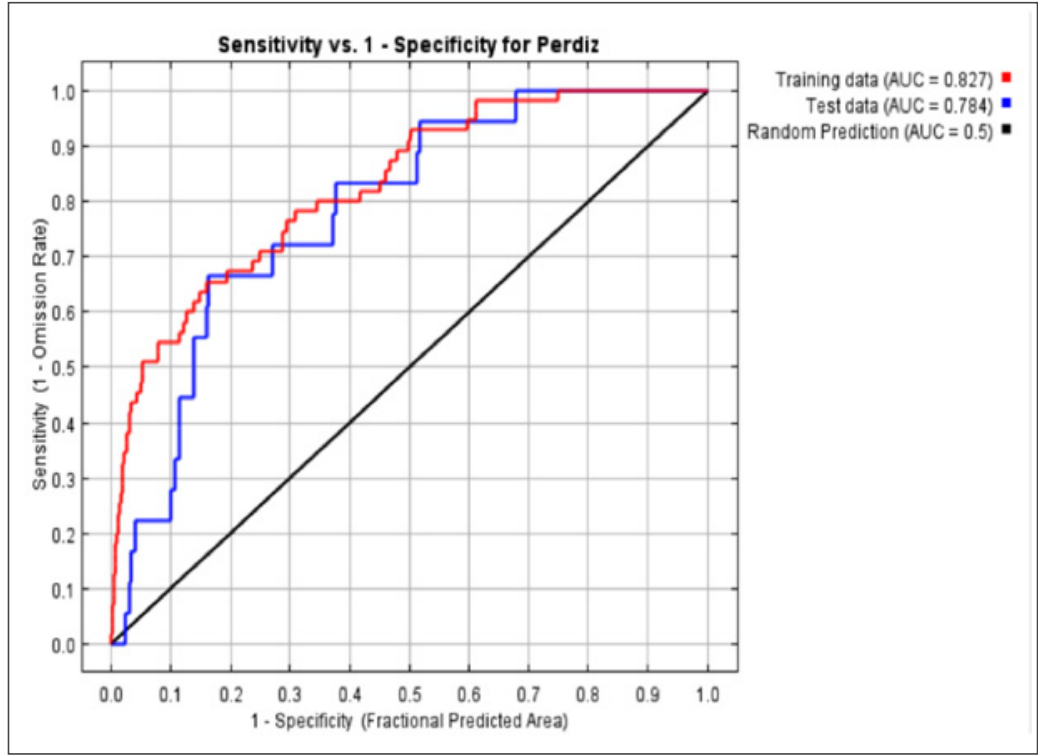

\subsection{Abundancia de Alectoris rufa}

Tras la reclasificación del modelo de hábitat se obtuvieron dos estratos de 5.475 ha y 1.283 ha de superficie cada uno de ellos. Los valores de densidad y abundancia calculados con estos dos estratos fueron un $20 \%$ inferiores a los valores obtenidos mediante el cálculo realizado considerando el área de estudio un sólo estrato, siguiendo el modo tradicional (Tabla 3). Además, mediante la utilización de los estratos se obtuvo estadísticamente un coeficiente de variación menor al obtenido para el cálculo de un sólo estrato, y unos intervalos de confianza del $95 \%$ más próximos al valor medio (Tabla 3), lo que indica que la estimación es más precisa. En todo caso, las densidades medias obtenidas se situaron por encima de la densidad máxima estimada en la Península Ibérica de 100 perdices por cada 100 ha (Blanco Aguiar et al. 2004).

\begin{tabular}{lccc}
\hline Tipo de cálculo & $\begin{array}{c}\text { Densidad } \\
\text { (perdices/ha) }\end{array}$ & \% C V & $\begin{array}{c}\text { Abundancia (no } \\
\text { de perdices) }\end{array}$ \\
\hline Densidad 1 (calculada en un solo estrato) & 1,297 & 33,05 & 8.766 \\
Densidad 2 (2 estratos modelo Maxent) & 1,033 & 23,93 & 6.983 \\
\hline
\end{tabular}




\subsection{Evolución temporal de la abundancia de Alectoris rufa}

A partir del modelo calculado con Maxent obtuvimos la distribución de estratos reflejada en la Figura 4 para los años 2000 y 2011, observándose ligeras diferencias entre ambos años. A pesar de que estas diferencias observadas no resultan muy llamativas, supusieron una reducción del 30\% de la superficie de mayor densidad (estrato A) en el periodo 2000 - 2011. En consecuencia, y tras aplicar las densidades obtenidas en el apartado anterior para cada uno de los estratos, se estimó que la población de Alectoris rufa habría pasado durante este periodo de los 8.213 ejemplares estimados para el año 2000 a los 6.983 ejemplares estimados en la actualidad. Esta reducción en la población estaría provocada según el modelo del hábitat por los cambios producidos en la composición y configuración del paisaje.

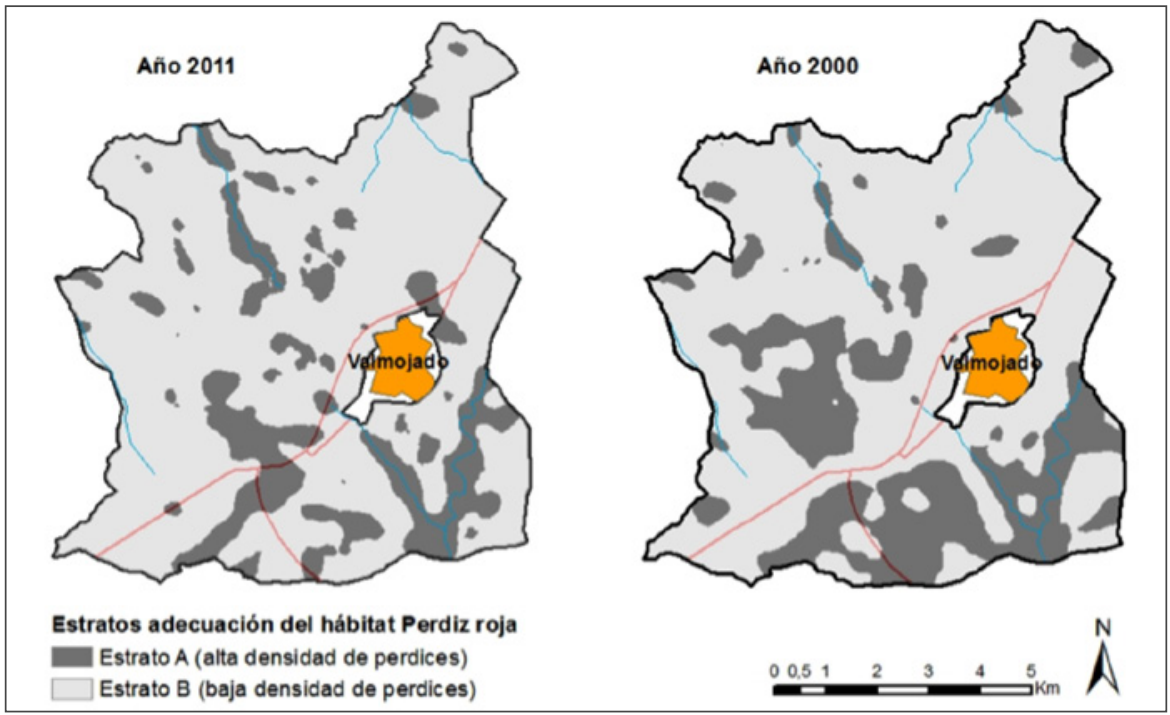

\section{DISCUSIÓN}

\subsection{Modelos predictivos de hábitat y estimación de la abundancia de Alectoris rufa}

La interpretación ecológica del modelo de hábitat apoya las conclusiones obtenidas en estudios previos realizados sobre la especie Alectoris rufa, basando su predicción en 9 variables de las cuales 6 son índices que representan la estructura del paisaje. En este sentido, Herranz et al. (2000) y Fortuna (2002) determinan que esta especie selecciona zonas de cultivo con matorral fragmentado, en el que abundan eriales, coscojares, aulagares y tomillares, adquiriendo gran importancia la abundancia de linderos ocupados por vegetación natural. Más recientemente, Buenestado et al. (2008) describe que las perdices evitan zonas 
con vegetación leñosa y zonas totalmente desnudas, mientras que seleccionan positivamente áreas cultivadas, áreas con una cobertura de vegetación media, y prados o praderas. En relación a estos estudios, nuestro modelo coincide en una selección positiva de las áreas de cultivo fragmentadas, con alta densidad de parches, y evitando la existencia extensas superficies dedicadas a un único uso. Dentro de este tipo de estructura se observó una favorabilidad hacia los cultivos de viñedo, y un ligero efecto desfavorable hacia los pastizales, siendo indiferente para el resto de ocupaciones. Este rechazo sobre las zonas de pastizal se opone a lo descrito por Fortuna (2002), que determina que los eriales formados por tierras de cultivo abandonadas confieren al paisaje una componente muy importante en la selección del hábitat para las perdices. No obstante, los pastizales tratados en este estudio representan en su gran mayoría tierras agrícolas de cereal y viñedo abandonadas recientemente, las cuales han sido colonizadas por vegetación arvense que al no ser pastoreada alcanza una importante cobertura vegetal de hasta unos $50 \mathrm{~cm}$ de altura, confiriéndoles unas características muy particulares y diferentes a los eriales descritos en Fortuna (2002). Por otro lado, también se dedujo del modelo que las perdices seleccionan positivamente las zonas de fondos de valle frente a las zonas de cerro o de la ladera, aunque se trata de un efecto que no ha sido analizado en otros estudios similares en combinación con las variables de estructura del paisaje.

En cuanto a los valores de abundancia obtenidos, se observa un coeficiente de variación y unos intervalos de confianza menores en el cálculo realizado por estratos que en el de un único estrato, lo que indica que la estimación obtenida es más precisa en el primero de los casos.

La abundancia obtenida a partir del modelo de hábitat fue un $20 \%$ menor a la obtenida sin su utilización, por lo que se deduce que mediante el procedimiento de estimación de un sólo estrato se estaría sobredimensionando el tamaño de la población de Alectoris rufa. Esto hace que se considere relevante la utilización de los modelos de distribución en el cálculo de densidades con el fin de mejorar la precisión en los resultados ajustándolos lo máximo posible a la realidad. No obstante, aunque la relación entre probabilidad de ocurrencia y abundancia podría ser válida para algunas especies y niveles de densidad, en ocasiones dicha relación no se cumple, por lo que no puede ser asumida directamente (Nielsen et al. 2005). En consecuencia es necesario seguir investigando en ese tema y, por tanto, es necesario tener precaución a la hora de aplicar esta metodología de estimación de abundancia a otras especies o ecosistemas.

La utilización de esta metodología en la estimación de densidades, además de aportar una posible mejora en la precisión de la estimación nos permite realizar proyecciones y predecir los efectos que podría causar sobre la abundancia una determinada acción de transformación del ecosistema. Por tanto, se trata de una herramienta que puede ser muy útil en labores de gestión, permitiéndonos valorar a través de predicciones los efectos de una determinada actuación sobre las especies y sus ecosistemas.

\section{Efecto de la transformación del paisaje sobre la abundancia de Alectoris rufa}

La proyección del modelo de hábitat llevado a cabo para el año 2000 mostró que durante los últimos años se ha producido una disminución de la superficie que reúne las condiciones más adecuadas para soportar una alta densidad de perdices, lo que habría provocado una merma en la abundancia de la especie Alectoris rufa en el área de estudio. 
Teniendo en cuenta que las únicas variaciones aportadas para la realización de esta proyección fueron los cambios producidos sobre la ocupación del suelo, se deduce que esta disminución de la abundancia de Alectoris rufa es consecuencia directa de estos cambios territoriales. No obstante existe la posibilidad de que otro tipo de factores no tenidos en cuenta en el modelo, como el cambio en las condiciones climáticas o las interacciones bióticas, también podrían tener su influencia en la variación de la abundancia de Alectoris rufa. En cuanto a los cambios territoriales analizados, la ocupación del suelo se ha visto transformada como consecuencia de cambios en la actividad agraria, pasando de tierra de labor a viñedo o viceversa, y principalmente como consecuencia del abandono de cultivos agrícolas, tanto tierras de labor como viñedos. La variación que en el conjunto del área de estudio ha supuesto el primero de los aspectos se puede considerar insignificante, pero por el contrario, el abandono de tierras agrícolas sí ha tenido una incidencia considerable durante los últimos años afectando aproximadamente al 20\% del área total de estudio. Ejemplos similares de estas transformaciones las podemos encontrar en áreas relativamente próximas (Romero-Calcerrada \& Perry 2004) si bien generando otros efectos ambientales negativos.

Según el modelo de hábitat obtenido los ejemplares de Alectoris rufa no seleccionan los terrenos de pastizal (los cuales se corresponden a estas tierras agrícolas abandonadas), lo que a priori estaría provocando la disminución en la abundancia de Alectoris rufa. Sin embargo, la distribución espacial obtenida y la interpretación ecológica del resto de variables indican que tiene más relevancia la modificación del mosaico agrario y la pérdida de superficie de viñedo que la propia proliferación de pastizales en sí. Esto es debido fundamentalmente a la homogeneización de la ocupación de la tierra que se ha producido en aquellas áreas donde el abandono agrícola se ha llevado a cabo de una forma más generalizada.

Algunos autores coinciden en que la intensificación de la agricultura es la causante de la disminución de las poblaciones de Alectoris rufa (Fortuna 2002 y Vargas 2002). Sin embargo en nuestro caso de estudio pensamos que un nuevo factor -el abandono de la actividad agraria-podría ser causante de la disminución de abundancia de Alectoris rufa. Estas causas que aparentemente son contradictorias indican que lo importante es la conservación del sistema tradicional de agricultura para sostener altas densidades de Alectoris rufa, manteniendo un mosaico con variedad de cultivos donde aparecen entremezclados parches de matorral y abundan los linderos con vegetación natural entre parcelas (Jiménez-García et al. 2006, Buenestado et al. 2008 y Belda et al. 2011). Esto quiere decir que tanto la intensificación como el abandono producen efectos negativos sobre las poblaciones de Alectoris rufa, siendo recomendable establecer adecuados planes de gestión que favorezcan la conservación de los mosaicos agrarios e impidan el abandono de tierras agrícolas.

\section{CONCLUSIONES}

La utilización de modelos de distribución de especies como complemento en la estimación de densidades y abundancia es una alternativa para tratar de precisar las estimaciones realizadas. Además, esta metodología nos permite realizar predicciones sobre los efectos que puede causar una determinada actuación sobre la abundancia de la especie censada, lo que le confiere gran utilidad en labores de gestión cinegética del territorio. 
Por otro lado, la densidad de Alectoris rufa que hemos obtenido en esta zona es de las más altas de la Península Ibérica, obteniéndose los máximos bajo las siguientes características óptimas del hábitat: existencia de valles, alta densidad de parches, ausencia de parcelas mayores a 70 ha, y presencia en el mosaico de cultivos de parcelas de viñedo. Por consiguiente, el abandono de viñedos y de tierras de labor está provocando una disminución de la abundancia de Alectoris rufa en la zona.

Como resultado de todo lo anterior, si queremos preservar las poblaciones de Alectoris rufa es necesario la implantación de medidas que favorezcan el mantenimiento de la actividad agrícola bajo unas condiciones tradicionales que garanticen su estructura de mosaico. Además, el mantenimiento de este tipo de hábitat favorecería la conservación y asentamiento de otras especies sensibles para las cuales la especie Alectoris rufa supone un importante recurso trófico, como ocurre en el caso de la mayoría de las rapaces presentes en la zona: Accipiter gentilis (azor común), Hieraaetus pennatus (aguililla calzada), Hieraaetus fasciatus (águila perdicera), Aquila chrysaetos (águila real); de mamíferos como el Vulpes vulpes (zorro rojo), Mustela nivalis (comadreja), Genetta genetta (gineta), y de algunas especies de reptiles que se alimentan de huevos y pollos durante la época de reproducción.

\section{BIBLIOGRAFÍA}

ARAÚJO, M.B. \& LUOTO, M. (2007). «The importance of biotic interactions for modelling species distributions under climate change». Global Ecology and Biogeography, 16: 743-753.

ARAÚJO, M.B., \& NEW, M. (2007). «Ensemble forecasting of species distributions». Trends in Ecology and Evolution, 22(1): 42-47.

BELDA, A., MARTÍNEZ -PÉREZ, J.E., PEIRO, V., SEVA, E., ARQUES, J. (2011). «Main landscape metrics affecting abundance and diversity of game species in a semi-arid agroecosystem in the Mediterranean region». Spanish Journal of Agricultural Research, 9(4): 1197-1212.

BENITO DE PANDO, B. \& PEÑAS DE GILES, J. (2007). «Aplicación de modelos de distribución de especies a la conservación de la biodiversidad en el sureste de la península ibérica». International Review of Geographical Information Science and Technology, GeoFocus, 7: 100-119.

BILLETER, R., LIIRA, J., BAILEY, D., BUGTER, R., ARENS, P., AUGENSTEIN, I., AVIRON, S., BAUDRY, J., BUKACEK, R., BUREL, F., CERNY, M., DE BLUST, G., DE COCK, R., DIEKÖTTER, T., DIETZ, H., DIRKSEN, J., DORMANN, C., DURKA, W., FRENZEL, M., HAMERSKY, R., HENDRICKX, F., HERZOG, F., KLOTZ, S., KOOLSTRA, B., LAUSCH, A., LE COEUR, D., MAELFAIT, J. P., OPDAM, P., ROUBALOVA, M., SCHERMANN, A., SCHERMANN, N., SCHMIDT, T., SCHWEIGER, O., SMULDERS, M.J.M., SPEELMANS, M., SIMOVA, P., VERBOOM, J., VAN WINGERDEN, W.K.R.E., ZOBEL, M. AND EDWARDS, P.J. (2008). «Indicators for biodiversity in agricultural landscapes: a pan-European study». Journal of Applied Ecology, 45: 141-150.

BLANCO AGUIAR, J.A., VIRGÓS, E., VILLAFUERTE, R. (2004). «Perdiz Roja, Alectoris rufa» en Madroño, A., González, C., Atienza, J.C. (Eds.). Libro Rojo de las Aves de España: 182-185. Dirección General para la Biodiversidad - Sociedad Española de Ornitología, Madrid. 
BORRALHO, R., REGO, F., VAZ PINTO, P. (1996). «Is driven transect sampling suitable for estimating red-legged partridge Alectoris rufa densities?» Wildlife Biology, 2:4: 259-268.

BUCKLAND, S.T., ANDERSON, D.R., BURNHAM, K.P., LAAKE, J.L. BORCHERS, D.L., THOMAS, L. (2001). Introduction to distance sampling. Oxford University Press, Oxford, United Kingdom.

BUEHLER, E.C. \& UNGAR, L.H. (2001). «Maximum Entropy Methods for Biological Sequence Modelling». BIOKDD, 60-64

BUENESTADO, F.J., FERRERAS, P., DELIBES-MATEOS, M., TORTOSA, F.S., BLANCO-AGUILAR, J.A., VILLAFUERTE, R. (2008). «Habitat selection and home range size of red-legged partridges in Spain». Agriculture, Ecosystems and Environment, 126: $158-162$.

DIRECCIÓN GENERAL DEL CATASTRO (2011). «Cartografía vectorial». Disponible en la página web: www.catastro.meh.esELITH, J.H., GRAHAM, C.P., ANDERSON, R.P., DUDIK, M., FERRIER, S., GUISAN, P., HIJMANS, R.J., HUETTMANN, F., LEATHWICK, J.R., LEHMANN, A., LI, J., LOHMANN, L.G., LOISELLE, B.A., MANION, G., MORITZ, C., NAKAMURA, M., NAKAZAWA, Y., OVERTON, J.M., PETERSON, A.T., PHILLIPS, S.J., RICHARDSON, K., SCACHETTI-PEREIRA, R., SCHAPIRE, R.E., SOBERÓN, J., WILLIAMS, S., WISZ, M.S., ZIMMERMANN, N.E. (2006). «Novel methods improve prediction of species distributions from occurrence data». Ecography, 29(2): 129-151.

ELITH, J.H., PHILLIPS, S.J., HASTIE, T., DUCÍK, M., CHEE, Y.E., YATES, C.J. (2011). «A statistical explanation of Maxent for ecologist». Diversity and Distributions, 17: 43-57.

EMLEN, J.T. (1977). «Estimating breeding season bird densities from transects counts». The Auk, 92: 455-468.

ENGLER, R., GUISAN, A., RECHSTEINER, L. (2004). «An improved approach for predicting the distribution of rare and endangered species from occurrence and pseudoabsence data». Journal of Applied Ecology, 41: 263-274.

FORTUNA, M.A. (2002). «Selección de hábitat de la Perdiz roja Alectoris rufa en periodo reproductor en relación con las características del paisaje de un agrosistema de la mancha (España)». Ardeola, 49(1): 59-66.

GUISAN, A. \& ZIMMERMANN, N.E. (2000). «Predictive habitat distribution models in ecology». Ecological modelling, 135: 147-186.

HERRANZ, J., YANES, M., SUAREZ, F. (2000). «Relaciones entre la abundancia de las especies de caza menor, sus depredadores y la estructura del hábitat en Castilla-La Mancha (España)». Ecología, 14: 219-233.

HIRZEL, A.H., HAUSSER, J., CHESSEL, D., PERRIN, N. (2002). «Ecological niche factor analysis: How to compute habitat-suitability maps without absence data?» Ecology, 83: 2027-2036.

JENNESS, J. (2006). «Topographic position index». Jenness Enterprises. Disponible en la página web: http://www.jennessent.com/arcview/tpi.htm.

JIMÉNEZ-GARCÍA, D., MARTÍNEZ-PÉREZ, J.E., PEIRÓ, V. (2006). «Relationship between game species and landscape structure in the SE of Spain». Wildlife Biology in Practice, 2(2): 48-62. 
LUCIO, A. \& PURROY, F. J. (1992). «Red-legged Partridge (Alectoris rufa) habitat selection in northern Spain». Gibier Faune Sauvage, 9: 417-429.

MATEO, R.G., FELICÍSIMO, A.M., MUÑOZ, J. (2011). «Modelos de distribución de especies: Una revisión sintética». Revista Chilena de Historia Natural, 84: 217-240.

MCGARIGAL, K., CUSHMAN, S.A., NEEL, M.C., ENE, E. (2002). «Fragstats: Spatial Pattern Analysis Program for Categorical Maps». Computer software program produced by the authors at the University of Massachusetts, Amherst. Disponible en la página web: http://www.umass.edu/landeco/research/fragstats/fragstats.html

MINISTERIO DE FOMENTO (2011). «Plan Nacional de Ortofotografía Aérea». Disponible en la página web: http://centrodedescargas.cnig.es/CentroDescargas/index.jsp

NIELSEN, S.E., JOHNSON, C.J., HEARD, D.C., BOYCE, M.S. (2005). «Can models of presence-absence be used to scale abundance? Two case studies considering extremes in life history». Ecography, 28: 197-208.

PEARCE, J.L. \& BOYCE, M.S. (2006). «Modelling distribution and abundance with presence-only data». Journal of Applied Ecology, 43: 405-412.

PEARCE, J.L. \& FERRIER, S. (2000). «Evaluating the predictive performance of habitat models developed using logistic regression». Ecological Modelling, 133: 225-245.

PHILLIPS, S.J., ANDERSON, R.P., SCHAPIRE, R.E. (2006). «Maximum entropy modeling of species geographic distributions». Ecological Modelling, 190: 231-259.

PÔÇAS, I., CUNHA, M., PEREIRA, L. (2011). «Remote sensing based indicators of changes in a mountain rural landscape of Northeast Portugal». Applied Geography, 31: 871-880.

RIVAS MARTÍNEZ, S. (1987). Mapa de las Series de Vegetación de la Península Ibérica. ICONA. Ministerio de Agricultura Pesca y Alimentación, Madrid.

ROMERO-CALCERRADA, R. \& LUQUE, S. 2006. «Habitat quality assessment using Weights-of-Evidence based GIS modelling: The case of Picoides tridactylus as species indicator of the biodiversity value of the Finnish forest». Ecological Modelling, 196: 62-76.

ROMERO-CALCERRADA, R., \& PERRY, G. L. W. 2004. «The role of land abandonment in landscape dynamics in the SPA 'Encinares del rio Alberche y Cofio, Central Spain, 1984-1999». Landscape and Urban Planning 66: 217-232.

SÉRGIO, C., FIGUEIRA, R., DRAPER, D., MENEZES, R., SOUSA, A.J. (2007). «Modelling bryophyte distribution based on ecological information for extent of occurrence assessment». Biological Conservation, 135(3): 341-351.

SHANNON, C.E. (1948). «A Mathematical Theory of Communication». The Bell System Technical Journal, 27: 379-423, 623-656.

STOCKWELL, D \& PETERS, D. (1999). «The GARP modelling system: problems and solutions to automated spatial prediction». International Journal of Geographical Information Science, 13, 2: 143-158.

TAPIA, L. \& DOMÍNGUEZ, J. (2007). «Broad-scale habitat use by red-legged partridge (Alectoris rufa) in a low-density area in northwestern Spain». European Journal of Wildlife Research, 53: 178-182.

TELLERÍA, J.L. (1986). Manual para el censo de los vertebrados terrestres. Raíces, Madrid. THOMAS, L., BUCKLAND, S.T., REXSTAD, E.A., LAAKE, J.L., STRINDBERG, S., HEDLEY, S.L., BISHOP, J.R.B., MARQUES, T.A., BUMHAM, K.P. (2010). «Distance 
software: design and analysis of distance sampling surveys for estimating population size». Journal of Applied Ecology, 47: 5-14.

VARGAS, J.M. (2002). Alerta cinegética. Reflexiones sobre el futuro de la caza en España. Otero, Madrid.VARGAS, J.M., GUERRERO, J.C., FARFÁN, M.A., BARBOSA, A.M., REAL, R. (2006). «Land use and environmental factors affecting red-legged partridge (Alectoris rufa) hunting yields in southern Spain». European Journal of Wildlife Research, 52: 188-195. 